\title{
Analysis of Signal Saturation in a Fiber Ring Resonator integrating an Intensity Sensor
}

\author{
Regina Magalhães ${ }^{\mathrm{a}}$, Susana O. Silva ${ }^{\mathrm{a}}$, Orlando Frazão ${ }^{\mathrm{a}}$
}

aINESC TEC and Department of Physics and Astronomy, Faculty of Sciences of University of Porto, Rua do Campo Alegre 687, 4169-007 Porto, Portugal (phone: (+351) 220402 301; fax: (+351) 220 402 437;

\begin{abstract}
The proposed technique consists in an optical fiber resonator interrogated for sensor characterization, implementing an alternative technique for dynamic range improvement. Such technique relies on the analysis of an added-signal caused by signal saturation, which occurs due to the broadening of the laser pulse. A wide study for different pulse widths is presented in this work, namely for $100 \mathrm{~ns}, 5 \mu \mathrm{s}$ and $20 \mu \mathrm{s}$, being the last one related to the emergence of an added-signal for the proposed configuration. The behavior of the waveform in the presence of an intensity sensor is also characterized.
\end{abstract}

Keywords: Fiber Ring Resonator, Added-signal, Saturation, Sensor

\section{INTRODUCTION}

Since the appearance of the optical fiber couplers it was possible to develop new types of optical devices, such as fiber loop mirrors, ring resonators and cavity ring-down devices. The incorporation of ring resonators is very wide, particularly in the sensors field [1-3]. The implementation of conventional interrogation systems, such as Optical Time Domain Reflectometers (OTDRs) in particular, allows the user to interrogate ring resonators in reflection [4-5]. Other techniques also integrate OTDRs in their interrogation systems, such as Cavity ring-down ones [6]. More recently, it was demonstrated the added-signal in a cavity ring-down configuration, presenting increased sensitivity when comparing to the conventional technique [7]. The added-signal had shown to increase optical power, increasing, as well, the ring-down time due to the sum of the several loops that light travels inside the ring.

In this work, is presented a wide study showing the behavior of the output signal, which is made varying the input parameters, namely the pulse width. The pulses studied are $100 \mathrm{~nm}, 5 \mu$ s and $20 \mu \mathrm{s}$. The behavior with applied displacement is also studied with an intensity sensor for each pulse width.

\section{EXPERIMENTAL RESULTS}

The experimental setup of the proposed optical system is represented in Figure 1. The cavity is composed by a standard $3 \mathrm{~dB}$ optical fiber coupler $(2 \times 2)$, a fiber loop with $\sim 500 \mathrm{~m}$ (SMF 28) and a sensing device characterized by a fiber tied in the shape of an eight number figure, which induces loss in fiber. A commercial OTDR, operating at $1550 \mathrm{~nm}$, is used as a light source to send impulses down into the fiber ring, instead of the usual laser and modulator setup in similar techniques [1]. The interrogation system is formed by a photodetector with $20 \mathrm{~dB}$ internal gain connected to an oscilloscope. Initially, the OTDR operates as a modulated multimode laser transmitter that sends a squared pulse of light down into the singlemode fiber. This train of pulses is coupled via the $3 \mathrm{~dB}$ arm of the input port of the ring resonator; it circulates around the fiber loop, being then coupled out via the $3 \mathrm{~dB}$ arm of the output port, going toward the photodetector. This system causes an amplitude decay of 50\% with time of the output pulses and some other attenuation due to the total existing losses in the fiber loop such as fiber coupler insertion losses, fiber loss and the sensor transmission attenuation. Only the first pulse does not suffers a 50\% amplitude decay, since it is directly interrogated by the photodetector and the oscilloscope without passing through the fiber ring. Although studying the saturation of the output signal, it should be noted that in this type of configuration there is no dependence of the output amplitude in relation to the power of the input signal, so that there is only output signal dependence with time and the features of the cavity. The advantage of using this equipment is to allow system optimization either in terms of impulse and optical power - allowing its use in long distance applications. The main 
goal of this implemented technique is to get a thorough study of the saturation regime for this optics, changing the parameters from the source drive.

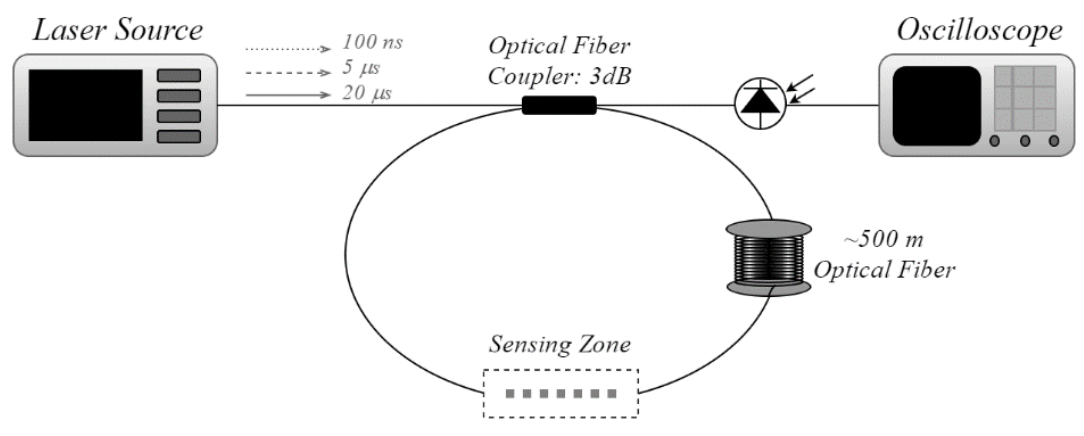

Figure 1. Experimental configuration of the proposed system operating with an intensity sensor.

Hence, the pulse width was initially set at $100 \mathrm{~ns}$ for a span range of $100 \mathrm{~km}$, and in a subsequent phase at $5 \mu$ s for a span range $512 \mathrm{~km}$. The waveform of the two different pulses is represented in Figure 2. As one can see, the shortest test pulse does not overload the reading system providing a non-saturated trace which does not compromise the oscilloscope's ability to identify the events. One can clearly see distinct pulses, separated by a measured cavity round trip time of $5.7 \mu \mathrm{s}$, which corresponds to the time that light takes to travel inside of the ring. This type of waveform is typical in conventional configurations [2]. On the other hand, besides leading to traces with better performances in terms of distance capability and less noise by the OTDR, a larger pulse can considerably decrease this ability when interrogated by the photodetector and the oscilloscope. In this specific case, the $5 \mu$ s pulse saturates the signal, which becomes a series of squared pulses slightly apart in time. This phenomena occurs because the pulse width was set very close to the cavity round trip time (5.7 $\mu \mathrm{s})$, which means that part of a given pulse arrives to the oscilloscope very nearly to the next pulse.

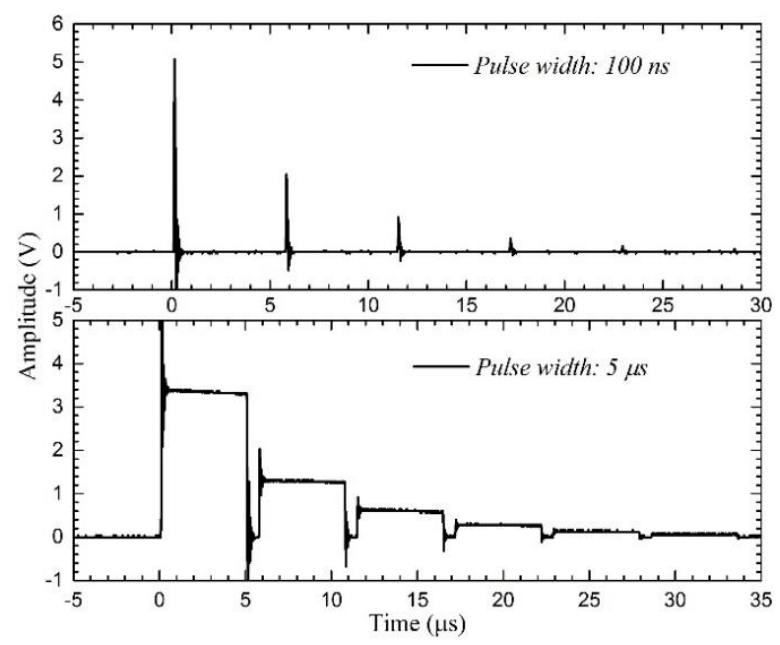

Figure 2. Waveforms of the proposed technique, obtained for different test pulse widths.

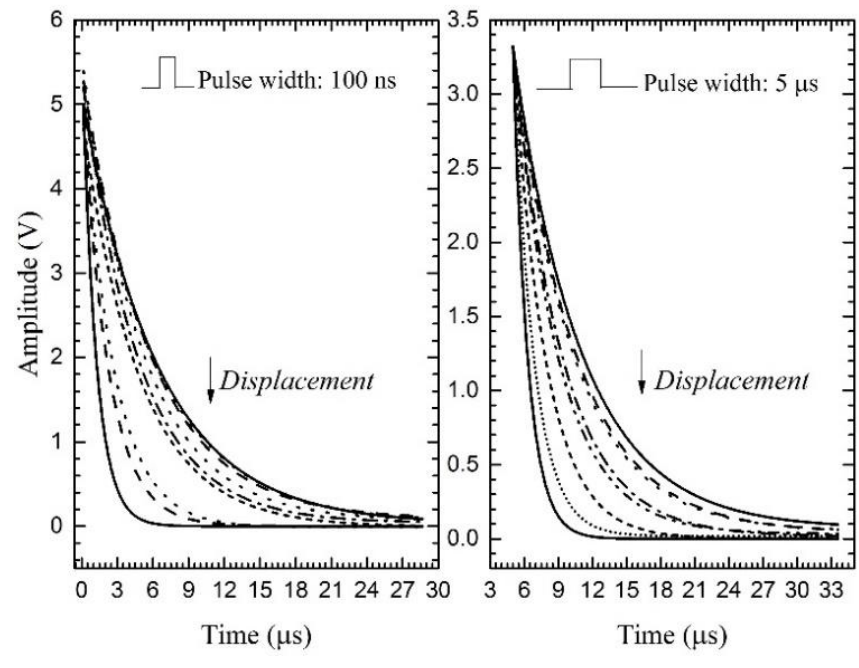

Figure 3. - Relation between the amplitude of peaks with applied displacement and time for (a) $100 \mathrm{~ns}$ and (b) $5 \mu$ s pulse width.

In order to obtain comparisons between the $100 \mathrm{~ns}$ and the $5 \mu$ s pulse, a fiber tied in the shape of an eight number figure in fiber was used as a displacement sensor. The displacement, $L$, was applied via sequential $50 \mathrm{~mm}$ displacements and measured according to one of the arms of the sensor, while the other one remained fixed. Figure 3 represents the relation between the amplitude of the output peaks during time, showing the exponential fits performed to the data. Table 1 shows the results attained for three different displacement measurements. 
Table 1. Decay times of displacement sensor with different pulses.

\begin{tabular}{ccc} 
Displacement & $\begin{array}{c}\text { Decay Time } \\
(\mathbf{1 0 0} \mathbf{~ n s})\end{array}$ & $\begin{array}{c}\text { Decay Time } \\
(\mathbf{5} \boldsymbol{\mu s})\end{array}$ \\
\hline $400 \mathrm{~mm}$ & $4.32 \mu \mathrm{s}$ & $3.99 \mu \mathrm{s}$ \\
$550 \mathrm{~mm}$ & $3.27 \mu \mathrm{s}$ & $3.00 \mu \mathrm{s}$ \\
$700 \mathrm{~mm}$ & $1.51 \mu \mathrm{s}$ & $1.27 \mu \mathrm{s}$ \\
\hline
\end{tabular}

From the results in Table 1, one can conclude that, in general, the $5 \mu$ s pulse presents lower decay times for high, medium or low displacements; however the amplitude presents similar behaviors with similar decay times for both cases. Previously, it was observed that a test pulse width close to the cavity round trip time causes saturation of the signal, as shown in Figure 2. Therefore, another pulse width was studied and set at $20 \mu \mathrm{s}$.

The behavior of this structure as a displacement sensor was also characterized using the $20 \mu$ pulse width. Results in Figure 5 show decreasing values of the amplitudes of the squared pulses, eventually reducing to a point where the signal obtained converges to a big squared pulse, where no distinction between pulses is observed. The trace attained for amplitude can be separated in two distinct parts: the first one with respect to the growth curve, where the more intense pulses are added-up; the other one with respect to the decay curve, caused by the low amplitude of the pulses that are being added-up after a few passages through the ring. Setting intermediate points in the middle of the pulses, the two curves were characterized through exponential fits (see Figure 5).

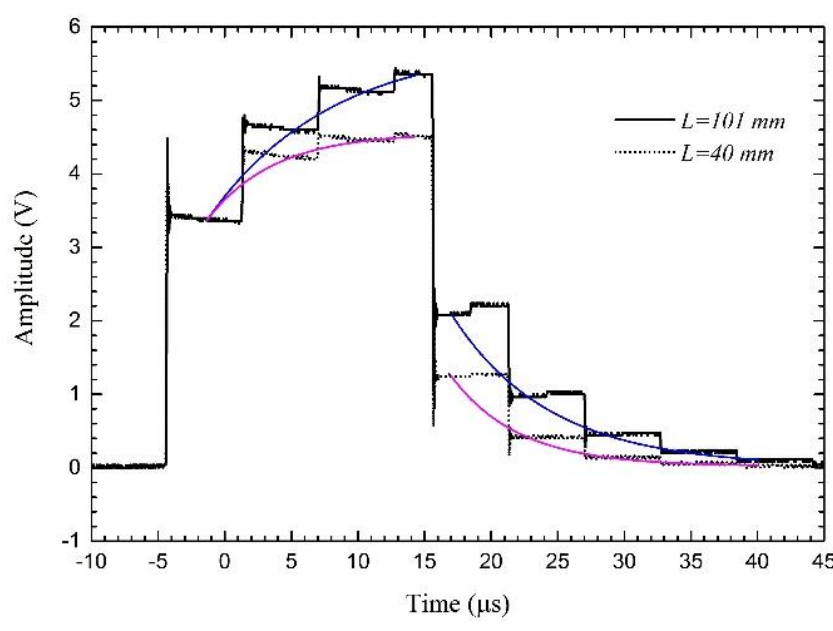

Figure 5. - Added-signal at $L=101 \mathrm{~mm}$ and $L=40 \mathrm{~mm}$. Four exponential fits were performed, for the rising and decaying points.

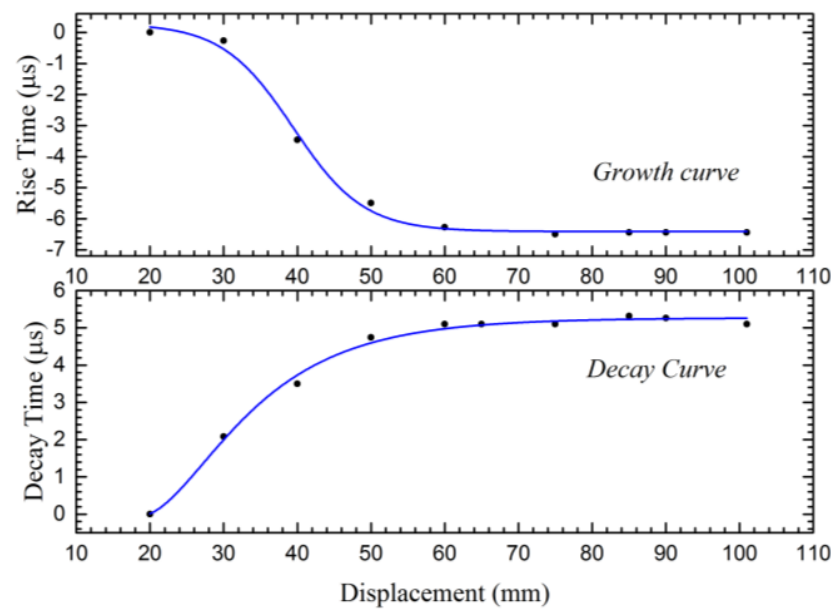

Figure 6. - Relation between the rise and decay times with the applied displacement.

Using the type of analysis presented in Figure 5, a study was performed considering the rising times (regarding the growth curves) and the decay times (concerning the decay curves). The relation between these times and the displacement applied to the sensor is represented in Figure 6. According to these two Figures, a greater displacement is associated with bigger rise and decay times.

Comparing the three decay times obtained for each displacement value, one can analyze that before the addition of the signal, the shortest impulse $(100 \mathrm{~ns})$ is related to bigger decay times, whereas the largest pulse $(5 \mu \mathrm{s})$ is associated to lower decay times. Although, the values for both cases are actually close to the same displacement value. On the other hand, the difference is much more distinguishable when studying a pulse with a larger width than the cavity round trip time. In this configuration, the decay time values are bigger than the two previous cases, which indicates that after the addition of the signal a larger pulse can be associated with greater times, reverting the behavior expected in the previous case. Since these were the results with greater decay times, the implementation of very large pulses can be benefic in some types of optical configurations, such as cavity ring-down ones, where bigger decay times are intended. Nonetheless, as lower decay times 
offer less sensitivity in terms of measurements, the growth curves could be used to improve the sensitivity of the system, since, in this case, the rise times are set in a larger range, offering more sensitivity.

\section{CONCLUSIONS}

It was proposed a wide analysis of signal saturation in a Fiber Ring Resonator, integrating an intensity sensor for displacement sensing. From the results, it was observed that a test pulse width close to the cavity round trip time causes saturation of the signal. Comparing results for $100 \mathrm{~ns}$ and for $5 \mu$ s given a cavity round trip time of $5.7 \mu \mathrm{s}$, it was observed that the shortest impulse was related to greater decay times, although the values for both cases were close, to the same displacement value. When comparing to the $20 \mu \mathrm{s}$ pulse, it was observed two distinct behaviors, yielding to a growth curve and a decay curve. Analyzing the respective obtained times, it was shown that the previous behavior was reversed, since this larger pulse was associated with the larger decay time obtained. With greater decay and rise times for greater displacements, this alternative technique enabled the improvement of dynamic range when integrated in the proposed setup. Therefore, this technique can be valuable when integrated in some types of setups, such as cavity ring-down ones.

\section{AKNOWLEDGMENT}

This work was financed by the FCT, Fundação para a Ciência e Tecnologia (Portuguese Foundation for Science and Technology) and by the ERDF (European Regional Development Fund) through COMPETE Programme (Operational Programme for Competitiveness) within project FCOMP-01-0124-FEDER-037281. S. O. Silva received a Pos-Doc fellowship (ref. SFRH/BPD/92418/2013) also funded by the EU and the Portuguese State

\section{REFERENCES}

1. Meyer, R. E., et al. "Passive fiber-optic ring resonator for rotation sensing." Optics letters 8.12 (1983): 644-646.

2. Küng, A., et al. "Optical fiber ring resonator characterization by optical time-domain reflectometry." Optics letters 22.2 (1997): 90-92.

3. Capmany, José, and Miguel A. Muriel. "Double-cavity fiber structures as all optical timing extraction circuits for gigabit networks." Fiber \& Integrated Optics 12.3 (1993): 247-255.

4. Küng A., J. Budin, L. Thévenaz and P. A. Robert "Rayleigh fiber optics gyroscope," IEEE Photonics Technology Letters, 9.7 (1997): 973.

5. Magalhães, R., S. O. Silva, and O. Frazão. "Fiber ring resonator using a cavity ring-down interrogation technique for curvature sensing." Microwave and Optical Technology Letters 58.2 (2016): 267-270.

6. Passos, D. J., et al. "Fiber cavity ring-down using an optical time-domain reflectometer."Photonic Sensors 4.4 (2014): 295-299.

7. Silva, S., et al. "Fiber-Optic Cavity Ring Down Using an Added-Signal for Curvature Sensing." Photonics Technology Letters, IEEE 27.19 (2015): 2079-2082. 